\title{
Bone Scintigraphy and Tenofovir-Induced Osteomalacia in Chronic Hepatitis B
}

\author{
Alex Khoo Cheen $\mathrm{Hoe}^{1} \cdot$ Lee Yeong Fong ${ }^{1}$
}

Received: 30 September 2015 / Revised: 24 December 2015 / Accepted: 7 January 2016/Published online: 26 January 2016

(C) Korean Society of Nuclear Medicine 2016

\begin{abstract}
Tenofovir, used in the treatment of chronic hepatitis B and HIV, is known for its side effects on the kidneys and bones. We share interesting images of a patient with tenofovir-induced osteomalacia on Technetium-99 m hydroxymethyelene (Tc-99 m HDP) bone scintigraphy. Pattern recognition of this bone
\end{abstract}

scintigraphy and correlation with the clinical history is essential to avoid misdiagnosis.

Keywords Hepatitis B · Osteomalacia · Tc-99 m HDP . Tenofovir

Alex Khoo Cheen Hoe

dr.alexkhoo@gmail.com

1 Department of Nuclear Medicine, Penang Hospital, Jalan Residensi, 10990 Georgetown, Penang, Malaysia 


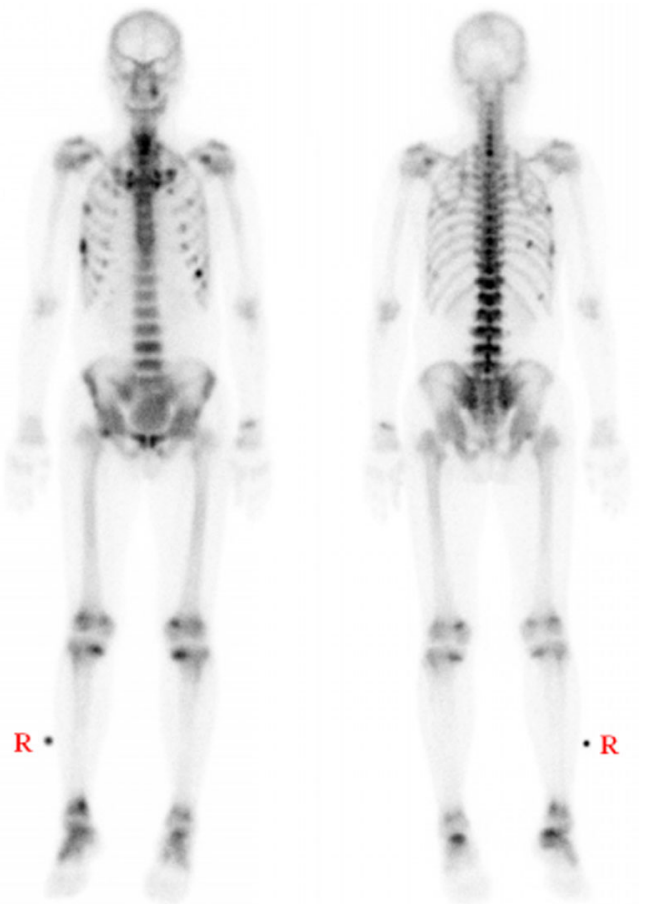

Fig. 1 A 51-year-old gentleman with chronic hepatitis B was prescribed tenofovir disoproxil fumarate one year ago. He had no other significant medical history and was relatively well until the last nine months ago when he developed persistent back pain. Clinical examination in early September 2015 revealed no significant abnormalities. However, he was noted to have hypophosphataemia, hypouricemia, raised creatinine level, and raised alkaline phosphatase level. His corrected calcium and other liver function tests were within normal limits. Hepatitis B DNA viral load was low. Ultrasound scan findings of the liver and kidneys were normal. Dual energy x-ray absorptiometry (DEXA) scan showed osteoporotic changes. No obvious fractures were noted on plain chest radiography. Plain radiographs of the thoracolumbar spine showed osteopenic changes with reduced T12-L5 vertebral height. He was then referred for bone scintigraphy. Bone scintigraphy performed 3 hours post-injection of Technetium-99 m hydroxymethylene diphosphonate (HDP) reveals increased uptake of tracer in bilateral ribs, costochondral junctions, sternoclavicular joints, sternum, shoulders, along the thoracolumbar spines, pubic bones, sacroiliac joints, bilateral heads of the femur, right neck of the femur, bilateral knees, bilateral distal tibiae, and bilateral tarsal bones. The increased uptake at the ribs was likely due to pseudofractures. The kidneys were faintly visualized in this scan. The bone scintigraphy findings are consistent with metabolic bone disease and in this case, due to Tenofovir-induced osteomalacia. Tenofovir has been shown to cause Fanconi's syndrome where there is proximal tubular dysfunction causing impaired reabsorption of amino acids, urate, bicarbonate, and phosphate [1]. This in turns leads to increased excretion of these solutes into the urine. Since glomerular filtration is not affected by proximal tubule dysfunction, the creatinine level in this patient only showed relatively modest increment. Nevertheless, researchers have shown that the effect of Tenofovir could be seen up to 8 months after initiation of therapy and the effect can be accentuated in patients on protease inhibitors (e.g., ritonavir, indinavir, lopinavir) and/or taking non-steroidal anti-inflammatory drugs [2]. Withdrawal of Tenofovir may help to establish the cause and effect, and thus alleviate patient's condition [3]. This case highlights the importance of pattern recognition of such bone scintigraphy and correlation with the clinical history to avoid misdiagnosis
Acknowledgment The authors would like to thank the DirectorGeneral of Health Malaysia for the permission to publish this paper.

\section{Compliance with Ethical Standards}

Conflict of Interest Alex Khoo Cheen Hoe and Lee Yeong Fong declare that they have no conflict of interest.

Ethical Statement The study was approved by an institutional review board or equivalent and has been performed in accordance with the ethical standards laid down in the 1964 Declaration of Helsinki and its later amendments. The subject in the study gave written informed consent.

\section{References}

1. Magalhaes-Costa P, Matos L, Barreiro P, Chagas C. Fanconi syndrome and chronic renal failure in a chronic hepatitis $\mathrm{B}$ monoinfected patient treated with tenofovir. Rev Esp Enferm Dig. 2015;107:5124.

2. Hall AM, Hendry BM, Nitsch D, Connolly JO. Tenofovir-associated kidney toxicity in HIV infected patients: a review of the evidence. Am J Kidney Dis. 2011;57:773-80.

3. Martinez MVG, Gallardo FG, Pirogova T, Garcia-Samaniego J. Bone scintigraphy and secondary osteomalacia due to nephrotoxicity in a chronic hepatitis B patient treated with tenofovir. Rev Esp Med Nucl Imagen Mol. 2014;33:103-5. 